\title{
Iodine intakes in Irish children aged 5-12 years
}

\author{
E. Kane ${ }^{1}$, M. Buffini ${ }^{1}$, A. Nugent ${ }^{1,2}$, L. Kehoe ${ }^{3}$, J. Walton ${ }^{4}$, J. Kearney ${ }^{5}$, A. Flynn ${ }^{3}$ and \\ B. McNulty ${ }^{1}$ \\ ${ }^{1}$ UCD Institute of Food and Health, University College Dublin, Dublin, Ireland, \\ ${ }^{2}$ Institute for Global Food Security, Queen's University Belfast, Belfast, UK, \\ ${ }^{3}$ School of Food \& Nutritional Sciences, University College Cork, Cork, Ireland, \\ ${ }^{4}$ Department of Biological Sciences, Munster Technological University, Cork, Ireland and \\ ${ }^{5}$ Technological University Dublin, Dublin, Ireland
}

Iodine is an essential trace mineral with an integral physiological role in the production of thyroid hormones ${ }^{(1)}$. Normal thyroid function is essential for growth and neurodevelopment both in utero, and during childhood ${ }^{(2)}$. Thus, pregnant women and young children are considered the most vulnerable or 'at-risk' groups in terms of iodine deficiency ${ }^{(3)}$. There is limited information regarding iodine intakes by school-aged children in Ireland. Therefore, the aim of the current study was to estimate dietary iodine intakes from a nationally representative sample of 600 Irish children aged 5-12 years.

The food and beverage consumption data used in this analysis was collected by means of a four-day weighed food diary during the National Children's Food Survey II (NCFSII; 2017-2018) (www.iuna.net). The study was nationally representative of the Irish population apart from social class; thus, a statistical weighting factor was applied to account for differences. Dietary iodine intakes ( $\mu \mathrm{g} / \mathrm{d})$ were calculated using updated iodine concentration data obtained from the Irish total diet study ${ }^{(4)}$ and were expressed as median and interquartile ranges (IQR) using SPSSC V26.0. The percentage contributions of various food groups to overall iodine intake were investigated; in order to assess the influence of milk consumption specifically, participants were categorised into four groups: nonconsumers, low, medium, and high consumers of milk.

Median (interquartile range) iodine intakes in the total population were $107.3 \mu \mathrm{g} / \mathrm{d}(69.2,164.0 \mu \mathrm{g} / \mathrm{d})$. Significantly greater iodine intakes were observed in boys at $113.7 \mu \mathrm{g} / \mathrm{d}(75.3,171.4 \mu \mathrm{g} / \mathrm{d})$, compared to girls whose intakes were $99.4 \mu \mathrm{g} / \mathrm{d}(62.5,159.3 \mu \mathrm{g} / \mathrm{d})$ $(\mathrm{p}=0.010)$. In the population, $43 \%$ of participants did not meet the adequate intake of $90-120 \mu \mathrm{g} / \mathrm{d}$, recommended for their age group $^{(5)}$. When split by sex, $38 \%$ of boys and $48 \%$ of girls did not meet this recommendation. In contrast, $3.8 \%$ of the population exceeded the tolerable upper intake level of iodine for their age group ${ }^{(6)}$. The major source of iodine was milk, contributing to over $50 \%$ of iodine intakes. Other sources included breakfast cereals $(7 \%)$ and yogurts $(5 \%)$. A significant positive association was observed between milk consumption and dietary iodine intake, with non-consumers and low consumers of milk having significantly lower iodine intakes compared to medium and high milk consumers $(\mathrm{p}<0.001)$.

Whilst most Irish children in this study seem to have adequate dietary iodine intakes; the finding that milk is the predominant food source should be highlighted, as any changes in the production or consumption of milk could potentially have major implications for iodine intakes. Future research should consider assessing urinary iodine concentrations, a better indicator of iodine status and a continued review of dairy practices, milk consumption patterns and iodine intakes and status should be a priority going forward.

\section{Acknowledgements}

The study was funded by the Irish Department of Agriculture, Food and the Marine (DAFM) under the 2015 Food Institutional Research Measure (FIRM) awards. Many thanks to all the families who agreed to take part, the fieldworkers, and the staff of the National Children's Food Survey II.

\section{References}

1. Kapil U (2007) Sultan Qaboos Univ Med J 7, 267-272

2. Zimmermann MB (2009) Endocr Rev 30, 376-408

3. World Health Organization (2007) Geneva, Switzerland: World Health Organization

4. Food Safety Authority of Ireland (2016) Dublin, Ireland: Food Safety Authority of Ireland

5. European Food Safety Authority (2014) EFSA J 12,3660

6. Scientific Committee on Food (2002) Brussels, Belgium: European Commission 